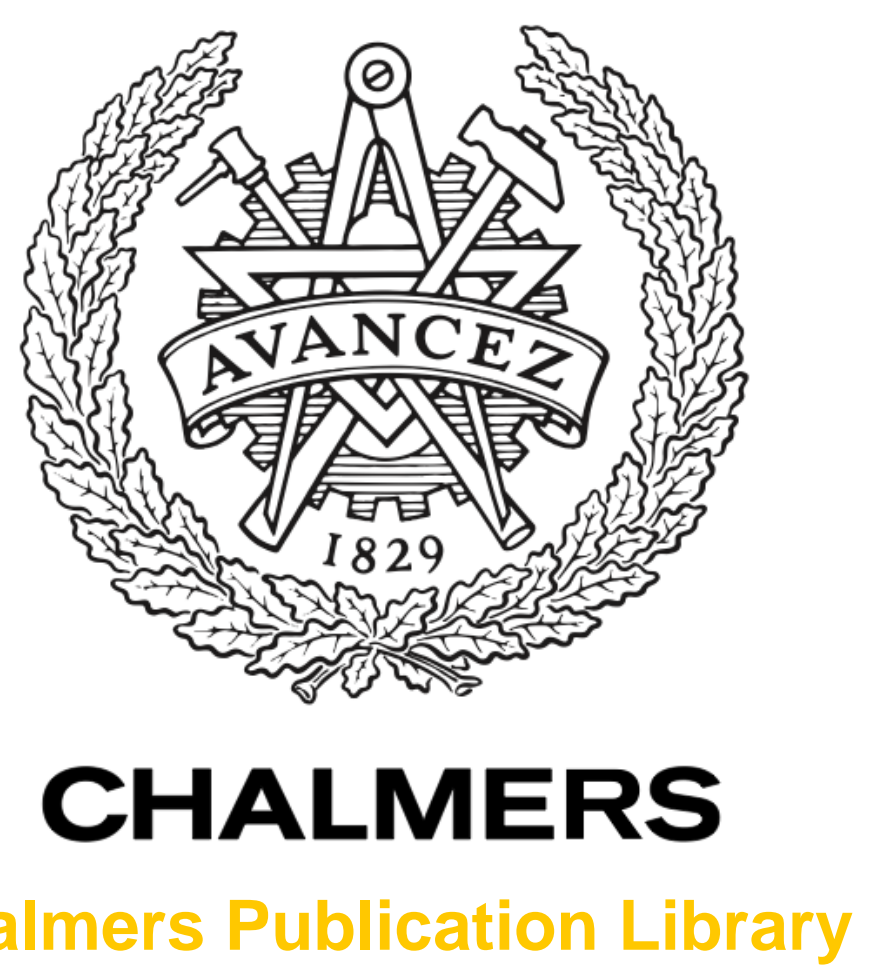

Chalmers Publication Library

\title{
Joint scheduling and localization in UWB networks
}

This document has been downloaded from Chalmers Publication Library (CPL). It is the author's version of a work that was accepted for publication in:

2015 IEEE International Conference on Communication Workshop, ICCW 2015

Citation for the published paper:

Garcia, G. ; Hu, W. ; Tay, W. et al. (2015) "Joint scheduling and localization in UWB networks". 2015 IEEE International Conference on Communication Workshop, ICCW 2015 pp. 724-729.

http://dx.doi.org/10.1109/ICCW.2015.7247267

Downloaded from: http://publications.lib.chalmers.se/publication/227679

Notice: Changes introduced as a result of publishing processes such as copy-editing and formatting may not be reflected in this document. For a definitive version of this work, please refer to the published source. Please note that access to the published version might require a subscription.

Chalmers Publication Library (CPL) offers the possibility of retrieving research publications produced at Chalmers University of Technology. It covers all types of publications: articles, dissertations, licentiate theses, masters theses, conference papers, reports etc. Since 2006 it is the official tool for Chalmers official publication statistics. To ensure that Chalmers research results are disseminated as widely as possible, an Open Access Policy has been adopted.

The CPL service is administrated and maintained by Chalmers Library. 


\title{
Joint Scheduling and Localization in UWB Networks
}

\author{
Gabriel E. Garcia*, Wuhua $\mathrm{Hu}^{\dagger}$, Wee Peng Tay ${ }^{\dagger}$, and Henk Wymeersch* \\ * Department of Signals and Systems, Chalmers University of Technology, Gothenburg, Sweden \\ $\dagger$ School of Electrical and Electronic Engineering, Nanyang Technological University, Singapore \\ E-mail: \{ggarcia,henkw\}@ chalmers.se, \{wptay,hwh\}@ntu.edu.sg
}

\begin{abstract}
Ultra-wide bandwidth (UWB) systems allow for accurate localization to tackle and complement the GPS-aided solutions that are impractical in weak signal environments. We consider the problem of fast link scheduling in the medium access control (MAC) layer for UWB localization. We present an optimization strategy to perform robust ranging scheduling with localization constraints. Given the complexity of the optimal strategy, two different MAC-aware link selection heuristics in terms of optimization strategies are also proposed. Our results show that significant MAC delay reductions are possible through the use of simple local heuristics.
\end{abstract}

\section{INTRODUCTION}

Absolute and relative position information is of crucial importance to present and future wireless networks, where location-aware applications and services are increasing in number at a rapid pace [1], [2]. Although Global Navigation Satellite Systems (GNSSs) can provide position information in many scenarios, they only provide outdoor coverage, and may not be feasible in certain conditions such as indoors, underground, or in urban canyons, where weak signal conditions are encountered [3]. As a result, there exists a need for accurate localization in scenarios where GNSS solutions are not viable.

UWB communication and ranging has been shown to be an up-and-coming technology to undertake the localization problem where GNSS is not available. In particular, pulsebased UWB offers specific advantages, including mitigation of small-scale fading and robustness against interference. Furthermore, considering a two-way time-of-arrival (TW-TOA) ranging procedure, accurate and precise ranging, proportional to the employed bandwidth, makes it a convenient technology for localization purposes [4].

UWB positioning systems have gathered significant attention in the research community, often with the specific aim of improving accuracy. Methods such as enhanced ranging [3], the employment of more anchor nodes, higher transmission powers [5], and cooperation among nodes [6], [7], [8] have shown to improve accuracy. However, these works do not consider the impact of the medium access control (MAC) layer within practical implementations. In the MAC layer, classical problems such as link scheduling and node scheduling have been extensively studied [9], giving rise to many centralized and distributed protocols. A well-known MAC approach is spatial reuse time division multiple access (STDMA), a collision-free scheme in which links (in our case ranging transactions) are allocated in the same time slot as long as they do not cause significant mutual interference [10]. The objective in STDMA link scheduling is to find the minimum number of time slots to schedule a given set of links in the network. A mathematical framework based on binary integer programming (BIP) and an efficient solution based on column generation is presented in [9]. However, scheduling methods for localization generally consider the set of links as a given, ignoring the coupling between scheduling and localization. As the MAC layer causes delays in the positioning systems that may adversely affect the overall performance, a joint design is appropriate.

The fundamental trade-off between localization accuracy and MAC delay was studied [11], but no practical schemes were proposed. In [12], [13] based on computer simulations authors investigate the cost delay poses to accuracy, for cooperative and target tracking, respectively. A decentralized self-stabilizing MAC protocol suitable for cooperative UWB navigation in multi-hop networks was proposed in [14], while a distributed and decentralized scheduling for cooperative localization was analyzed in [15] and [16]. Finally, focusing on the analysis and design of the MAC protocol in [17], a MAC design for cooperative localization networks was investigated.

In this paper, we establish an optimization framework for joint scheduling and localization, with the goal of allocating STDMA scheduling resources to perform UWB TW-TOA ranging with a target localization requirement. Our main contributions are as follows:

- We propose an optimization strategy for robust ranging scheduling with localization constraints, and include an approximation of the optimization problem into as an integer linear program (ILP); and

- We propose a collection of heuristics for MAC aware link selection, formalized as ILPs, which allow a decomposition of the original ILP into several local link selection subproblems and one centralized scheduling problem to alleviate the complexity issue imposd by the joint scheduling and localization.

Our results indicate that significant reductions in MAC delay are possible through simple local heuristics. 


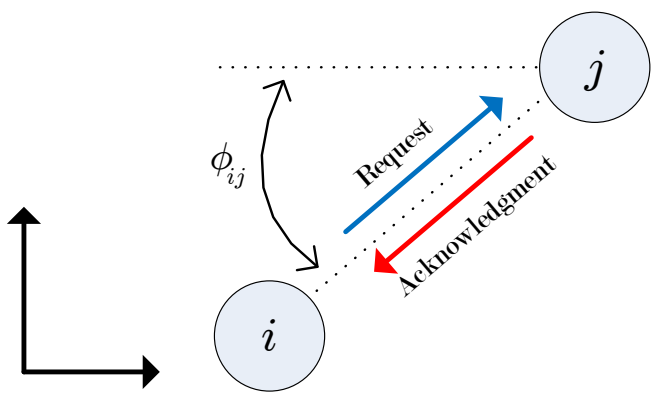

Figure 1. Two-dimensional graphic representation of the TW-TOA ranging transaction between nodes $i$ and $j$, where $\phi_{i j}$ is the angle between nodes with respect to the horizontal axis.

\section{SySTEM MODEL}

\section{A. UWB Localization}

We consider a sufficiently synchronous wireless network to enable TDMA scheduling consisting of a fusion center, $N$ agents, and $M$ anchor nodes, collected in the sets $\mathcal{S}_{\text {agents }}$ and $\mathcal{S}_{\text {anchors. }}$. Agents have unknown time-varying positions while anchors are nodes with known positions at all times. We focus on a specific time slot, where the two-dimensional position of node $i$ is denoted by $\mathbf{p}_{i}$, with an a priori distribution $p\left(\mathbf{p}_{i}\right)$. The angle with respect to the horizontal axis and the distance between nodes $i$ and $j$ are denoted by $\phi_{i j}$ (see Figure 1) and $d_{i j}$, respectively. The time slot duration $T$ is lower bounded by the measurement time $T_{\text {meas }}$, required by the agents to gather all the UWB measurements. The measurement time is divided up into shorter TDMA slots.

The measurements made by agent $i$ at the current time slot are denoted by $\mathbf{z}_{i}$. Measurements are obtained through TWTOA, whereby agent $i$ sends a request to anchor $j$, which responds back with an acknowledgment, depicted in Figure 1. Both nodes $i$ and $j$ estimate the TOA for request and acknowledgment, respectively. Agent $i$ uses the round-trip delay between nodes to calculate and estimate their distance. A TDMA time slot is needed per transaction, including request and acknowledgment. The measurement between agent $i$ and anchor $j$ is given by

$$
z_{i j}=d_{i j}+\frac{n_{i j}}{2}+\frac{n_{j i}}{2}
$$

where $d_{i j}=\left\|\mathbf{p}_{i}-\mathbf{p}_{j}\right\|$ is the Euclidean norm of the difference between the agent and anchors' two-dimensional position, $n_{i j}$ and $n_{j i}$ are the TOA errors of the request and acknowledgment between nodes. The errors are independent of each other and modeled as zero mean Gaussian random variables: $n_{i j} \sim \mathcal{N}\left(0, \sigma^{2}\right)$ and $n_{j i} \sim \mathcal{N}\left(0, \sigma^{2}\right)$. Under line-of-sight conditions, the ranging error variance between two nodes at a distance $d_{i j}$ apart can be modeled as in [6]:

$$
\sigma^{2}\left(d_{i j}\right)= \begin{cases}\sigma^{2} & d_{i j} \leq R_{\mathrm{hw}} \\ \sigma^{2} f\left(d_{i j}\right) & R_{\mathrm{hw}}<d_{i j} \leq R_{\max } \\ +\infty & d_{i j}>R_{\max },\end{cases}
$$

where $R_{\mathrm{hw}}$ is the range for which the variance is dominated by the hardware (e.g., ADC, filters), $R_{\max }$ is the maximum communication range, and $f\left(d_{i j}\right)$ is an exponential function with $f\left(R_{\mathrm{hw}}\right)=1$, capturing the ranging information intensity [5].

We aim to find the minimum number of time slots required to schedule ${ }^{1}$ all measurements within the network, constrained to a localization accuracy requirement, imposed by the application or service. Accuracy and MAC delay are evaluated using the squared position error bound (SPEB) [5] and the minimum required number of time slots to gather all information by the agents, respectively. The SPEB for agent $i$ is defined as

$$
\mathcal{P}_{i}=\operatorname{tr}\left\{\mathbf{J}_{i}^{-1}\right\},
$$

in which $\mathbf{J}_{i}$ is the $2 \times 2$ Fisher information matrix (FIM) of agent $i$. Assuming the set of edges in the network that are used for ranging transaction is denoted by $E$, then [5]

$$
\mathbf{J}_{i}=\sum_{j:(i, j) \in E} \frac{1}{\sigma^{2}\left(d_{i j}\right)} \mathbf{u}_{i j} \mathbf{u}_{i j}^{\mathrm{T}}
$$

where $\mathbf{u}_{i j}=\left[\begin{array}{lll}\cos \phi_{i j} & \sin \phi_{i j}\end{array}\right]^{\mathrm{T}}$.

\section{B. Network and MAC Model}

The wireless network consisting of $N+M$ nodes is represented by a communication graph $G=(V, E)$ with $V$ vertices (nodes) and the set $E=\left\{(i, j): i \in \mathcal{S}_{\text {agents }}, j \in \mathcal{S}_{\text {anchors }}\right\}$ representing the possible links between the nodes. We denote the power on link $(i, j)$ by $h_{i j}$, the transmit power by $P$, and the noise power by $W$. The STDMA MAC relies on the following rules:

1) an agent can only communicate with one anchor at a time;

2) an anchor can only communicate with one agent at a time;

3) an agent $i$ and anchor $j$ can communicate in the presence of other agent/anchor pairs (say $k$ and $l$ ), provided the following signal-to-interference-plus-noise-ratio (SINR) requirement is satisfied:

$$
\frac{P h_{i j}}{W+\sum_{k \neq i, l \neq j} P h_{k l}} \geq \gamma,
$$

where $\gamma$ is a suitable threshold. The SINR condition needs to hold both for the request $\left(\operatorname{SINR}_{i j}^{\mathrm{req}} \geq \gamma\right)$, and acknowledgment $\left(\operatorname{SINR}_{i j}^{\text {ack }} \geq \gamma\right)$ within the TW-TOA transaction.

${ }^{1}$ UWB is employed only for the measurement phase. Delays caused by agents transmitting information to the fusion center after data has been collected are not considered since these operations can be performed with an alternative radio technology. 


\section{JOINT SCHEDULING AND LOCALIZATION OPTIMIZATION FORMULATION}

This section develops the centralized joint scheduling and localization optimization strategy. The main goal is to minimize the number of time slots used to schedule TW-TOA transactions under a specific localization requirement and an STDMA scheme. We point out that the solver needs to have available the following information: the channel powers $h_{i j}$ for each pair of nodes, an estimate of the angles $\phi_{i j}$ and the distances $d_{i j}$. In practice, the channel powers are easily obtained through a short beaconing process. The angles and distances are inherently unknown (as we are solving a positioning problem), however reasonable estimates can be obtained through the a priori distributions $p\left(\mathbf{p}_{i}\right)$. In the absence of a priori knowledge, the proposed method will not be possible. More regarding the issue of a priori information will be discussed in Section V.a.

\section{A. Optimization Formulation}

The problem formulation follows a similar approach to the link formulation of a minimum-length scheduling with link oriented assignment (MLP) [18] . We divide up the positioning time $T$ into shorter TDMA slots indexed by $t$ and introduce the set $\mathcal{T}$, which contains the time slots employed to schedule ranging tansactions. For conciseness, we will call a TDMA time slot simply a time slot. We introduce variable $y_{t} \in\{0,1\}$ that defines whether or not a time slot $t$ is being used (i.e., $y_{t}=$ 1 if that the slot is used). In a similar manner $x_{i j t} \in\{0,1\}$, where $x_{i j t}=1$ if time slot is assigned to link $(i, j) \in E$ at time slot $t$. The optimization problem is posed as follows:

$$
\begin{aligned}
\mathcal{B}_{1}: \text { minimize } & \mathbf{y}^{\mathrm{T}} \mathbf{1} \\
\text { subject to } & x_{i j t} \leq y_{t}, \forall(i, j) \in E \\
& \sum_{t \in \mathcal{T}} x_{i j t} \leq 1, \forall(i, j) \in E \\
& \operatorname{SINR}_{i j t}^{\mathrm{req}} \geq \gamma, \forall(i, j) \in E \\
& \operatorname{SINR}_{i j t}^{\text {ack }} \geq \gamma, \forall(i, j) \in E \\
& \sum_{j:(i, j) \in E} x_{i j t} \leq 1, j \in \mathcal{S}_{\text {anchors }} \\
& \sum_{i:(i, j) \in E} x_{i j t} \leq 1, i \in \mathcal{S}_{\text {agents }} \\
& \sum_{t \in \mathcal{T}} \operatorname{tr}\left\{\mathbf{J}_{i}^{-1}\right\} \leq \alpha_{i}, \forall i \in \mathcal{S}_{\text {agents }} \\
& x_{i j t} \in\{0,1\}, \forall(i, j) \in E \\
& y_{t} \in\{0,1\} \\
& \forall t \in \mathcal{T}
\end{aligned}
$$

The objective (6a) aims to minimize the total number of time slots to schedule all the links ${ }^{2}$, where $\mathbf{y}$ collects $y_{t}$ for all $t \in \mathcal{T}$ and $\mathbf{1}$ is a vector containing ones of the the size as

\footnotetext{
${ }^{2}$ Slots for which the optimal $y_{t}=0$ can simply be discarded, thus reducing the schedule length.
}

y. Constraint (6b) states that a slot is considered as "used" if it is assigned to any link. Constraint (6c) specifies a requirement where every link may be assigned a slot within the schedule. Constraints (6d) and (6e) are the SINR requirements related to interference with concurrent transmissions for both the request and the acknowledgment within the TW-TOA ranging procedure. Similar as in [18], the SINR is constraints (6d) and (6e) are both explicitly denoted as:

$$
\begin{aligned}
& \operatorname{SINR}_{i j t}^{\text {req }}=\frac{h_{i j} x_{i j t} P+\left(1-x_{i j t}\right) \Omega}{W+\sum_{k \neq i, l \neq j} x_{k l t} h_{k j} P} \geq \gamma \\
& \mathrm{SINR}_{i j t}^{\mathrm{ack}}=\frac{h_{i j} x_{i j t} P+\left(1-x_{i j t}\right) \Omega}{W+\sum_{k \neq i, l \neq j} x_{k l t} h_{i l} P} \geq \gamma,
\end{aligned}
$$

where constant $\Omega>\gamma\left(W+\sum_{k \neq i, l \neq j} x_{k l t} h_{k j} P\right)$ with $x_{k l t}=1$ for all $k, l$ and $t$. Given that ranging transactions can only be initiated by agents, constraint (6f) prohibits transmission to the same anchor within the same time slot. Constraint $(6 \mathrm{~g})$ prevents each agent from performing more than one ranging procedure per time slot. The localization requirement in terms of the SPEB is given by (6h), where the SPEB for each agent should be smaller than a required threshold $\alpha_{i}$ (expressed in squared meters). The FIM for agent $i$ within the localization constraint is obtained from (4):

$$
\mathbf{J}_{i}=\sum_{j:(i, j) \in E} \frac{1}{\sigma^{2}\left(d_{i j}\right)} x_{i j t} \mathbf{u}_{i j} \mathbf{u}_{i j}^{\mathrm{T}}
$$

Finally, (6i) and (6j) force the variables to be binary. The problem $\mathcal{B}_{1}$ is an integer nonlinear problem due to constraint (6h) . In the next Section we will approximate it with an integer linear program.

\section{B. ILP Approximation based on Greshgorin's Circle Theorem}

Problem $\mathcal{B}_{1}$ is nonlinear due to the localization constraint (6h). We replace the nonlinear constraint (6h) with a linear constraint, ensuring that it is still satisfied. We focus on a specific agent and will drop the index $i$. The FIM $\mathbf{J}$ is always positive semi-definite, with eigenvalues, say $\lambda_{1} \geq 0$ and $\lambda_{2} \geq$ 0 . We can express (6h) equivalently as

$$
\lambda_{1}^{-1}+\lambda_{2}^{-1} \leq \alpha .
$$

One way to satisfy (10) is as follows

$$
\begin{aligned}
& \lambda_{1} \geq 2 / \alpha \\
& \lambda_{2} \geq 2 / \alpha .
\end{aligned}
$$

According to Greshgorin's circle theorem [19] , the eigenvalues $\lambda_{1}$ and $\lambda_{2}$ must lie in the following intervals

$$
\begin{aligned}
& \lambda_{1} \in\left[\left|J_{11}\right|-\left|J_{12}\right|,\left|J_{11}\right|+\left|J_{12}\right|\right] \\
& \lambda_{2} \in\left[\left|J_{22}\right|-\left|J_{21}\right|,\left|J_{22}\right|+\left|J_{21}\right|\right]
\end{aligned}
$$

where $J_{m n}$ are the entry of $\mathbf{J}$ on row $m$, column $n$. Thus, to guarantee a required localization error, the eigenvalues need to be larger than the lower bound, i.e., $\left|J_{11}\right|-\left|J_{12}\right|$ and $\left|J_{22}\right|-$ 


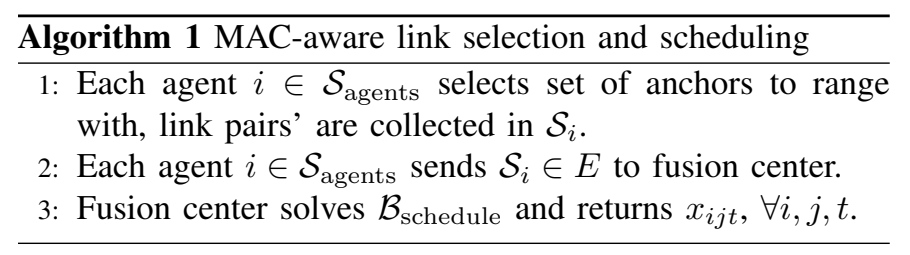

$\left|J_{21}\right|$, for $\lambda_{1}$ and $\lambda_{2}$, respectively. This allows us to rewrite the constraint as follows:

$$
\begin{aligned}
\sum_{t \in \mathcal{T}} \sum_{j:(i, j) \in E} x_{i j t} c_{i j} s_{i j} & \leq \sum_{t \in \mathcal{T}} \sum_{j:(i, j) \in E} x_{i j t} c_{i j}^{2}-2 / \alpha_{i} \\
- & \sum_{t \in \mathcal{T}} \sum_{j:(i, j) \in E} x_{i j t} c_{i j} s_{i j} \leq \sum_{t \in \mathcal{T}} \sum_{j:(i, j) \in E} x_{i j t} c_{i j}^{2}-2 / \alpha_{i} \\
& \sum_{t \in \mathcal{T}} \sum_{j:(i, j) \in E} x_{i j t} c_{i j} s_{i j} \leq \sum_{t \in \mathcal{T}} \sum_{j:(i, j) \in E} x_{i j t} s_{i j}^{2}-2 / \alpha_{i} \\
- & \sum_{t \in \mathcal{T}} \sum_{j:(i, j) \in E} x_{i j t} c_{i j} s_{i j} \leq \sum_{t \in \mathcal{T}} \sum_{j:(i, j) \in E} x_{i j t} s_{i j}^{2}-2 / \alpha_{i},
\end{aligned}
$$

in which $c_{i j}=\cos \phi_{i j} / \sigma\left(d_{i j}\right)$ and $s_{i j}=\sin \phi_{i j} / \sigma\left(d_{i j}\right)$. By substituting (6h) with (15)-(18), all of which are linear in the optimization variables, we obtain a reformulation, say $\mathcal{B}_{2}$. This problem is in the form of an ILP and can be solved using standard solvers. Nevertheless, due to the large number of variables and constraints, problem $\mathcal{B}_{2}$ can only be solved from small networks.

\section{MAC-AWARE Link SELECTION AND SCHEDULing OPTIMIZATION FORMULATION}

In this section, we introduce two methods where we first perform link selection accounting for possible MAC bottlenecks, following by scheduling. These methods will be less complex than $\mathcal{B}_{2}$ and thus suitable to larger networks. The link selection subproblem can be executed on the individual agents and require each agent to solve a small ILP based on local network information, resulting in a set of links, say $\mathcal{S}_{i} \subseteq E$, which satisfy the localization constraints. Afterwards, the scheduling phase is solved in a centralized fashion, where the fusion center receives as input the set of links $\mathcal{S}_{i}$ for all $i \in \mathcal{S}_{\text {agents }}$ and returns a minimum length schedule based on the STDMA constraints. The process is depicted in Algorithm 1.

\section{A. Link Selection Subproblem}

Each agent $i$ selects the anchors to range with in order to attain the desired localization performance (defined by $\alpha_{i}$ ). The link selection is formulated as an ILP, where agent $i$ associates a cost $\Delta_{i j} \geq 0$ with each anchor $j$. The constraints in the ILP are the positioning accuracy constraints as in
Section III.b and are now of the form

$$
\begin{aligned}
& \sum_{j:(i, j) \in E} \omega_{j} c_{i j} s_{i j} \leq \sum_{j:(i, j) \in E} \omega_{j} c_{i j}^{2}-2 / \alpha_{i} \\
& -\sum_{j:(i, j) \in \mathcal{S}_{i}} w_{j} c_{i j} s_{i j} \leq \sum_{j:(i, j) \in E} \omega_{j} c_{i j}^{2}-2 / \alpha_{i} \\
& \sum_{j:(i, j) \in E} \omega_{j} c_{i j} s_{i j} \leq \sum_{j:(i, j) \in E} \omega_{j} s_{i j}^{2}-2 / \alpha_{i} \\
& -\sum_{j:(i, j) \in E} \omega_{j} c_{i j} s_{i j} \leq \sum_{j:(i, j) \in E} \omega_{j} s_{i j}^{2}-2 / \alpha_{i}
\end{aligned}
$$

so that the link selection ILP for agent $i$ becomes

$$
\begin{aligned}
\mathcal{B}_{\text {select }}^{(i)}: \text { minimize } & \sum_{j:(i, j) \in E} \omega_{j} \Delta_{i j} \\
\text { subject to } & (19)-(22), \forall i \in \mathcal{S}_{\text {agents }} \\
& \omega_{j} \in\{0,1\} \forall j:(i, j) \in E .
\end{aligned}
$$

We consider two ways of setting the costs $\Delta_{i j}$ : a one-hop approach and a two-hop approach.

1) One-hop link selection: for each anchor we initialize $\Delta_{i j}=1$. If for a certain anchor $d_{i j} \geq R_{\mathrm{hw}}$ or $P h_{i j} / W \leq \gamma$, we update $\Delta_{i j}=\theta$, where $\theta$ is a very large number. This avoids using anchors with poor connectivity to agent $i$ or poor ranging quality.

2) Two-hop link selection: for anchor we set $\Delta_{i j}$ as in the one-hop link selection. In addition, for each anchor $j$ and each agent $k \neq i$, we increment $\Delta_{i j}$ with 1 whenever $P h_{k j} \geq v W$, in which $v \geq 1$ is a parameter. This will assign a larger cost to anchors which have the potential to cause interference to other agents.

\section{B. Scheduling Subproblem}

The scheduling subproblem $\mathcal{B}_{\text {schedule }}$ is solved at the fusion center after each agent transmitted its own set of links $\mathcal{S}_{i} \subseteq E$ to be scheduled. The scheduling problem is posed similarly as $\mathcal{B}_{1}$, but without the FIM constraint (6h) and constraint (6c) adapted to include equality for links included in the union of all $\mathcal{S}_{i}$. Similar to [9], an efficient solution based on column generation method can be computed by means of a master and a restricted problem.

\section{RESUlts AND Discussion}

\section{A. A Priori Information}

As we have mentioned before, the values of $d_{i j}$ and $\phi_{i j}$ are not known, since they depend on the unknown positions. Given the distribution of the position of agent $i, p\left(\mathbf{p}_{i}\right)$ can be used to approximate $d_{i j}$ and $\phi_{i j}$. For instance when $\mathbf{p}_{i} \sim \mathcal{N}\left(\mathbf{m}_{i}, \sigma_{p, i}^{2} \mathbf{I}\right)$, we have $d_{i j} \approx\left\|\mathbf{m}_{i}-\mathbf{p}_{j}\right\|$ and similar for $\phi_{i j}$. The presence of a priori information can also be used to relax the constraint (6h) by adding $1 / \sigma_{p, i}^{2}$ to the right hand side of (15)-(18) or of (19)-(22). A priori information in practical scenarios can be obtained for example using proximity based localization or previous time step localization information, e.g., tracking. 


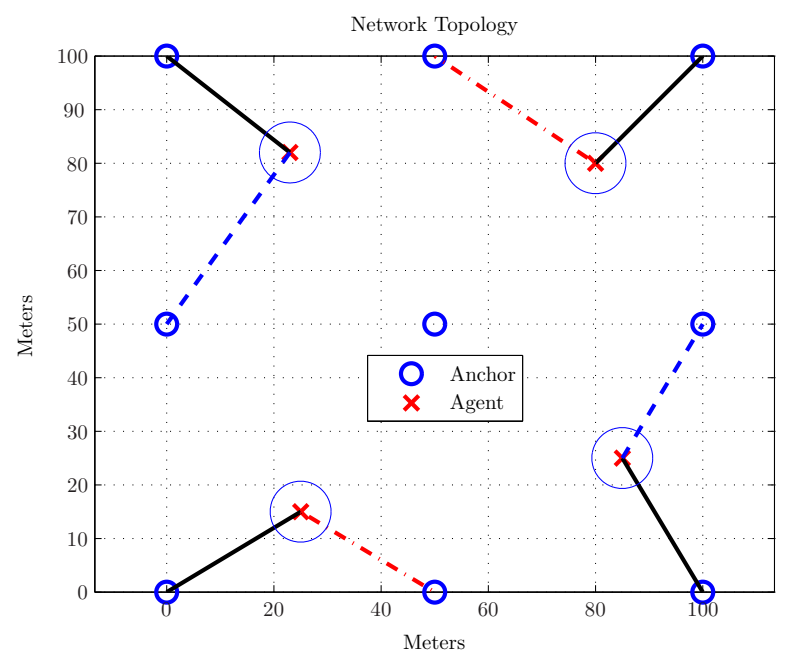

Figure 2. Example of optimal ranging sheduling for 9 anchors and 4 agents.

\section{B. Simulation Setup}

We consider a network topology in a square area of 100 $\mathrm{m} \times 100 \mathrm{~m}$ with anchors positioned in a similar fashion as in [6] while agents are uniformly distributed. The channel gain $h_{i j}=10^{\mathrm{PL}\left(d_{i j}\right) / 10}$ includes a simple path loss model $\operatorname{PL}\left(d_{i j}\right)=\operatorname{PL}\left(d_{0}\right)+10 \eta \log _{10}\left(d_{i j} / d_{0}\right)$, where $\operatorname{PL}\left(d_{0}\right)$ is the line-of-sight path loss at reference distance $d_{0}$ and $\eta$ is the path loss exponent. The simulation parameters used to obtain results are shown in Table I.

\begin{tabular}{|c|c|c|c|}
\hline Parameter & Value & Parameter & Value \\
\hline \hline$\eta$ & 3 & $W$ & $-20 \mathrm{dBm}$ \\
\hline$P$ & $10 \mathrm{~W}$ & $\sigma^{2}$ & $1 \mathrm{~m}^{2}$ \\
\hline$\gamma$ & $1.76 \mathrm{~dB}$ & $\sigma_{p, i}$ & $7 \mathrm{~m}$ \\
\hline$d_{0}$ & $1 \mathrm{~m}$ & $R_{h w}$ & $40 \mathrm{~m}$ \\
\hline$\alpha_{i}$ & $3 \mathrm{~m}^{2}$ & $v$ & 3 \\
\hline
\end{tabular}

Table I

EXAMPLE SIMULATION PARAMETERS.

\section{Simulation Results}

An example of a small network consisting of 9 anchors and 4 agents is depicted in Figure 2. The optimal schedule satisfying the localization and SINR constraints is computed from the optimization problem $\mathcal{B}_{2}$ and is shown graphically in the Figure 2. Around each agent, we show a $90 \%$ confidence ellipse containing the probable agents position with the use of the a priori information. The lines between anchors and agents represent the TW-TOA links to be scheduled. Links with the same format (line style) can be scheduled in the same time slot without without violating the SINR constraints. As we can observe, this specific network topology needs 3 time slots in order to perform the required measurements: 1 time slot for the 4 black solid links, 1 time slot for the 2 red dash-dotted links and 1 time slot for the two blue dashed links. As compared to a TDMA approach where 8 time slots would be required, the optimal solution shows only 3 time slots are needed, fulfilling both the localization requirement and the STDMA constraints.

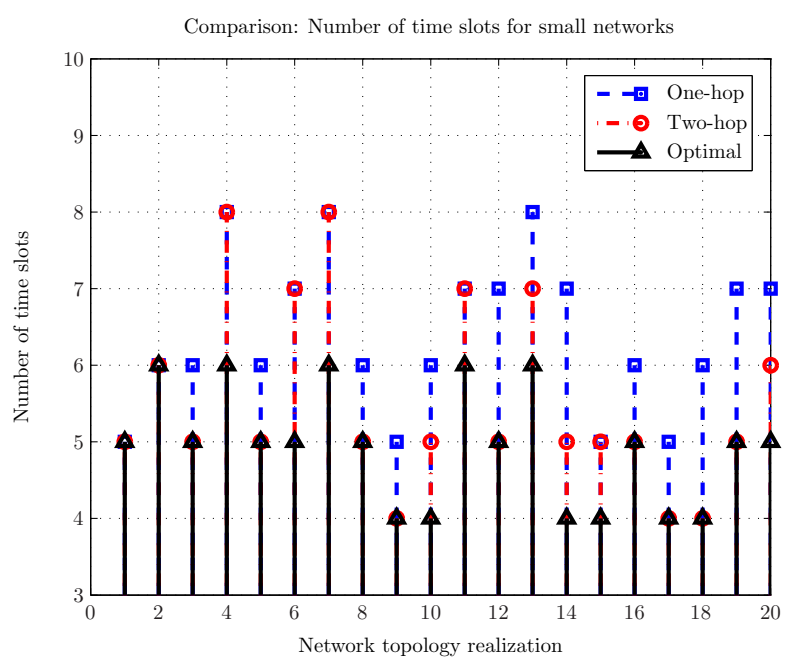

Figure 3. Number of time slots for 20 realizations consisting of 9 fixed anchors and 4 uniformly distributed agents.

Figure 3 shows 20 different realizations of network topologies consisting of 9 fixed positioned anchors placed in a similar manner as in [6] and 4 uniformly distributed agents within the $100 \mathrm{~m} \times 100 \mathrm{~m}$ square. In Figure 3 we can compare the required number of time slots for three different approaches: solutions to $\mathcal{B}_{2}, \mathcal{B}_{\text {select }}^{(i)}$ with one-hop link selection, and $\mathcal{B}_{\text {select }}^{(i)}$ with two-hop link selection. As mentioned in Section III.b, due to the large number of variables and constraints, problem $\mathcal{B}_{2}$ can only be solved for small network realizations. Thus, the comparison between the optimal solution and the two heuristic link selection methods is done with relatively small networks. From Figure 3, we can observe that the optimal solution shows the smallest number of time slots for each of the 20 realizations. The one-hop and two-hop heuristics perform similar, with the two-hop heuristic having a slightly better performance than the one-hop one, since its number of time slots is closer in most of the realizations to the optimal one. It is important to note that even if the one-hop and twohop heuristics result in suboptimal solutions, the complexity of the algorithm is decreased allowing for faster numerical computations.

We now consider larger networks and generate 20 different realizations of network topologies consisting of 13 fixed positioned anchors, once again, placed in a similar manner as in [6] and 8 uniformly distributed agent within the 100 $\mathrm{m} \times 100 \mathrm{~m}$ square. Given the complexity of the optimal formulation $\mathcal{B}_{2}$, it was excluded from the large network topology analysis. In Figure 4 we can observe than the twohop heuristic performs better since it required less number of time slots. From the simulations, it is observed that the twohop heuristic outperforms the one-hop one when there exist "bottleneck" anchors: anchors with several neighboring agents. The two-hop heuristic accounts for possible interfering agents within the cost $\Delta_{i j}$, alleviating the bottleneck anchor problem. 


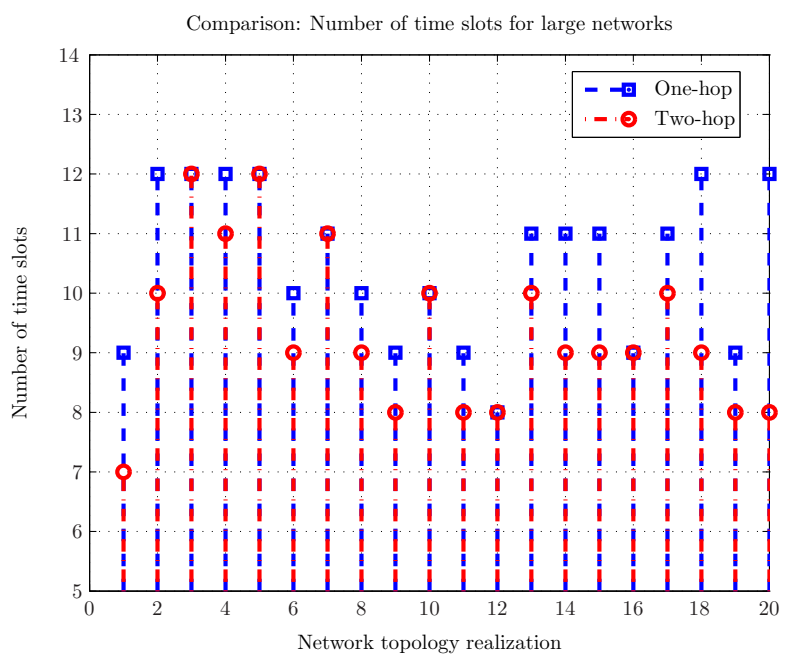

Figure 4. Number of time slots for 20 realizations consisting of 13 fixed anchors and 8 uniformly distributed agents.

\section{CONCLUSiOnS}

We have investigated the interplay between UWB localization accuracy and MAC delay. We have presented an optimization strategy for ranging scheduling with localization constraints and its approximation into an ILP to obtain a more efficient solution. We found that the optimal solution is not applicable for large scale networks, since its complexity grows very fast in the number of nodes in the network. In consequence, we presented two different approaches for MAC aware link selection: a one-hop and a two-hop heuristics, both formulated as ILPs . These approaches allow for decomposition of the original optimal ILP into several local link selection subproblems and once centralized scheduling problem. The decomposition shows to alleviate the complexity imposed by the original ILP with similar solutions in terms of the number of time slots required to perform ranging and attain a required localization performance. The two-hop link selection heuristic outperforms the one-hop counterpart when bottleneck anchors (anchors with many agents around) are present in the network, while both heuristic algorithms perform similar when the agent density is sparse.

Possible venues of future research include implementation in practical UWB networks, simulations with more realistic simulation parameters, and the use of cooperation among agents.

\section{ACKNOWLEDGMENT}

This research was supported in part, by the European Research Council, under Grant No. 258418 (COOPNET), the
Swedish Research Council, under Grant No. 2010-5889, and the Ericsson Research Foundation Grant FOSTIFT:14:066.

\section{REFERENCES}

[1] K. Pahlavan and X. Li, "Indoor geolocation science and technology," IEEE Communications Magazine, vol. 40, pp. 112-118, 2002.

[2] R. Di Taranto, S. Muppirisetty, R. Raulefs, D. Slock, T. Svensson, and H. Wymeersch, "Location-aware communications for 5g networks: How location information can improve scalability, latency, and robustness of 5G," Signal Processing Magazine, IEEE, vol. 31, no. 6, pp. 102-112, 2014.

[3] N. Patwari, J. N. Ash, S. Kyperountas, A. O. Hero III, R. L. Moses, and N. S. Correal, "Locating the nodes," IEEE Signal Processing Magazine, vol. 22, pp. 54-59, 2005.

[4] A. F. Molisch, P. Orlik, Z. Sahinoglu, and J. Zhang, "UWB-based sensor networks and the IEEE 802.15.4a standard - a tutorial," International Conference on Communication and Networking in China, 2006.

[5] Y. Shen and M. Z. Win, "Fundamental limits of wideband localization part I: A general framework," IEEE Transactions on Information Theory, vol. 56, pp. 4956-4980, 2010.

[6] H. Wymeersch, J. Lien, and M. Z. Win, "Cooperative localization in wireless networks," Proceedings of the IEEE, vol. 97, pp. 427-450, 2009.

[7] M. R. Gholami, S. Gezici, and E. G. Ström, "Improved position estimation using hybrid TW-TOA and TDOA in cooperative networks," IEEE Transactions on Signal Processing, vol. 60, pp. 3770-3785, 2012.

[8] Y. Shen, S. Mazuelas, and M. Z. Win, "Network navigation: Theory and interpretation," IEEE Journal on Selected Areas in Communications, vol. 30, pp. 1823-1834, 2012.

[9] P. Björklund, P. Värbrand, and D. Yuan, "A column generation method for spatial TDMA scheduling in adhoc networks," Ad Hoc Networks, vol. 2, no. 4, pp. 405-418, 2004.

[10] R. Nelson and L. Kleinrock, "Spatial TDMA: A collision-free multihop channel access protocol," IEEE Transactions on Communications, vol. COM-33, no. 9, pp. 934-944, 1985.

[11] G. Garcia, L. S. Muppirisetty, E. M. Schiller, and H. Wymeersch, "On the trade-off between accuracy and delay in cooperative UWB localization: Performance bounds and scaling laws," IEEE Transactions on Wireless Communications, vol. 13, no. 8, pp. 4574-4585, 2014.

[12] F. Sottile, A. Vesco, R. Scopigno, and M. Spirito, "MAC layer impact on the performance of real-time cooperative positioning," in IEEE Wireless Communications and Networking Conference (WCNC), 2012, pp. 18581863.

[13] C. Lindberg, L. S. Muppirisetty, K.-M. Dahlén, V. Savic, and H. Wymeersch, "MAC delay in belief concensus for distributed tracking," in 10th Workshop on Positioning, Navigation and Communication, 2013.

[14] P. Leone and E. M. Schiller, "Self-stabilizing TDMA algorithms for dynamic wireless ad-hoc networks," International Journal of Distributed Sensor Networks, vol. 2013, p. 17.

[15] T. Wang, Y. Shen, S. Mazuelas, and M. Z. Win, "Distributed scheduling for cooperative localization based on information evolution," IEEE International Conference on Communications, 2012.

[16] D. Satyam, D. Zachariah, A. De Angelis, and P. Handel, "Cooperative decentralized localization using scheduled wireless transmissions," IEEE Communications Letters, vol. 17, pp. 1240-1243, 2013.

[17] M. Rengasamy, E. Dutkiewicz, and M. Hedley, "MAC design and analysis for wireless sensor networks with co-operative localisation," International Symposium on Communications and Information Technologies, 2007.

[18] M. Cardei, I. Cardei, and D. Ding-Zhu, Resource Management in Wireless Networking, Springer, Ed., 2005.

[19] R. A. Horn and C. R. Johnson, Matrix Analysis. Cambridge University Press, 1987. 